\title{
TRANSFORMANDO EL SISTEMA DE INFORMACIÓN DE NACIMIENTOS EN EL PERÚ
}

\author{
Walter H. Curioso ${ }^{1, a}$, Karim Pardo ${ }^{1, b}$, Manuel Loayza ${ }^{1, c}$
}

\section{RESUMEN}

El Registro del Certificado de Nacido Vivo en Línea fue desarrollado gracias a un trabajo conjunto entre el Ministerio de Salud del Perú y el Registro Nacional de Identidad y Estado Civil. Se trata de un sistema gratuito que permite registrar a los recién nacidos en la misma sala de partos, y generar en tiempo real el certificado de nacido vivo, facilitando así el trámite necesario para la obtención del acta de nacimiento y del Documento Nacional de Identidad del recién nacido. Entre marzo de 2012 y marzo de 2013, este sistema se ha implementado en 114 establecimientos de salud, en 21 regiones, lo cual ha permitido el registro de 113917 recién nacidos vivos, y ha proporcionado información precisa, oportuna y de calidad sobre estos nacimientos. Es así que este sistema se constituye como un eje central del sistema de información en salud que permite obtener estadísticas en tiempo real para una adecuada y oportuna toma de decisiones en salud pública.

Palabra clave: Nacimiento vivo; Internet; Estadísticas; Atención primaria de salud (fuente: DeCS BIREME).

\section{TRANSFORMING THE PERUVIAN BIRTH INFORMATION SYSTEM}

\section{ABSTRACT}

The On-Line Registration of Certificates of Live Births was developed in conjunction with the Ministry of Health of Peru and the National Registry of Identification and Civil Status. It is a free system that registers newborns in the delivery room itself which generates a real time certificate of live birth. This simplifies the procedures required to obtain the birth certificate and the National Identity Document for the newborn. This system has been implemented in 114 health centers in 21 regions from March 2012 to March 2013, and has registered 113917 live births, providing accurate, timely and quality information about these births. This system is the main cornerstone of the health information system which helps obtain real time statistics for adequate and timely decision making in public health.

Key words: Live birth; Internet; Statistics; Primary health care (source: MeSH NLM).

\section{INTRODUCCIÓN}

Los registros de nacimientos y defunciones constituyen los principales componentes de las estadísticas vitales. La información producto de estos registros sirve de insumo importante para la toma de decisiones, y para el cálculo de estadísticas, análisis epidemiológicos y demográficos utilizados en el diseño, planificación y evaluación de programas y políticas en salud. Por ejemplo, las estadísticas vitales derivadas del análisis de los registros vitales sirven de fuente importante para la evaluación de los Objetivos de Desarrollo del Milenio ${ }^{(1)}$.

Sin embargo, la escasez de información confiable, oportuna, precisa y la falta de una apropiada codificación son desafíos que aún persisten en muchos estados. En muchos países en vías de desarrollo el registro de hechos vitales es incompleto, inoportuno, y con problemas en la calidad de datos. En la mayoría de los países subsaharianos y en algunos países de Asia la mayoría de las personas nacen y mueren sin ser reconocidos legalmente por sus gobiernos, y sin ser documentados en estadísticas oficiales (2). De acuerdo con el reporte de registro de nacimientos del Fondo de Naciones Unidas para la Infancia (UNICEF), el porcentaje de nacimientos que no se registran en regiones clave del mundo, varia notoriamente en función al grado de desarrollo de cada región, evidentemente pudiéndose apreciar valores más altos en países en vías de desarrollo. Es así que, en los países subsaharianos el porcentaje de nacimientos no registrados llega a $55 \%$, en comparación con un $15 \%$ encontrado en los países de América Latina y el Caribe, y un $2 \%$ en los países desarrollados ${ }^{(3)}$.

\footnotetext{
Oficina General de Estadística e Informática (OGEI), Ministerio de Salud. Lima, Perú.

a Médico, doctor en Informática Biomédica y magíster en Salud Pública; ${ }^{\mathrm{b}}$ médico, magíster en Salud Pública; ${ }^{\mathrm{c}}$ médico, magister Salud Pública, epidemiólogo. Recibido: 03-04-13 Aprobado: 08-05-13

Citar como: Curioso WH, Pardo K, Loayza M. Transformando el sistema de información de nacimientos en el Perú. Rev Peru Med Exp Salud Publica. 2013;30(2):303-7.
} 
Un sistema eficaz de registro de hechos vitales es aquel en el cual la recolección de datos no se realiza como sistema independiente, sino como parte de un sistema integrado de información (4). En ese sentido, con el desarrollo de las tecnologías de información y comunicación, el registro electrónico de hechos vitales se constituye en una herramienta importante para la recopilación continua de datos y para producir información de calidad para el cálculo de estadísticas vitales. En la actualidad, la mayoría de los datos disponibles sobre los programas de salud en el mundo provienen de métodos de estimación, censos, encuestas de hogares especializadas, o una combinación de todos estos métodos, en su mayoría, basados en papel ${ }^{(5)}$.

Las principales limitaciones de la mayoría de estos métodos se deben al hecho de que, a menudo, se implementan en áreas geográficas específicas 0 poblaciones específicas, limitando así su generalización a todo un país. Además, los actuales registros vitales basados en papel no permiten la recolección de datos de manera eficiente, y el proceso de obtención y análisis no es oportuno. En respuesta a esta situación, muchos países en el mundo están investigando soluciones eficaces para el rediseño del registro de hechos vitales como cimientos de los futuros sistemas nacionales de información en salud ${ }^{(6,7)}$.

En Perú, el Ministerio de Salud decidió fortalecer el sistema de información de nacimientos, desarrollando e implementando en marzo del 2012 una plataforma electrónica vía Internet para registrar a todo recién nacido en línea, inmediatamente posterior al nacimiento.

\section{DESARROLLO DEL SISTEMA DE INFORMACIÓN DE NACIMIENTOS EN LÎNEA}

El Registro del Certificado de Nacido Vivo en Línea fue desarrollado gracias al esfuerzo conjunto del Ministerio de Salud del Perú y el Registro Nacional de Identidad y Estado Civil (RENIEC). Su uso se oficializó con la Resolución Ministerial 148-2012/MINSA que aprueba la Directiva Administrativa 190-MINSA/OGEI-V01 que establece el Procedimiento para el Registro del Certificado de Nacido Vivo ${ }^{\left({ }^{8}\right)}$.

Se trata de un sistema gratuito que permite registrar a los recién nacidos en la misma sala de partos, y generar en tiempo real el certificado de nacido vivo, con lo cual se identifica al recién nacido desde su nacimiento, lo que a su vez facilita el trámite necesario para obtener su acta de nacimiento y su Documento Nacional de Identidad (DNI), además de facilitar el acceso oportuno al Sistema Integral de Salud y a los programas sociales que ofrece el estado.

El sistema funciona en la misma sala de partos, y el establecimiento solo debe contar con una computadora con acceso a Internet, necesaria para la instalación del aplicativo a través del cual se accede al Registro del Certificado de Nacido Vivo en Línea. El personal de salud, inmediatamente después del parto, registra los datos de la madre, ingresa el número de su DNI, se conecta a la base de datos del RENIEC y verifica la información. Luego se registran los datos del recién nacido, como sexo, peso, talla, y se registran los datos del profesional de la salud que participó de la atención del parto. Esta información es validada en tiempo real con la base de datos del RENIEC. El término de la atención se da con la emisión del Certificado de Nacido Vivo, el cual se completa con la impresión de la huella del pie del recién nacido y la huella dactilar de la madre.

\section{IMPLEMENTACIÓN DEL SISTEMA DE INFORMACIÓN DE NACIMIENTOS EN LÍNEA}

El proceso de implementación del Sistema de Información de Nacimientos fue diseñado considerando una fase de selección de los establecimientos de salud. Para esta selección se tomó en cuenta una distribución de Pareto, con la cual se determinó los establecimientos de salud que concentran el $80 \%$ de partos atendidos, $y$ otras variables como conectividad a Internet y establecimiento en el cual se ubica un punto de digitación del Seguro Integral de Salud. Posteriormente, se coordinó con las direcciones regionales de salud para evaluar los parámetros de accesibilidad geográfica y su cercanía a capitales de provincia, con el fin de identificar la coincidencia con los puntos registrales de RENIEC.

Luego de consensuar los establecimientos de salud con las direcciones regionales de salud, se inició la priorización de las capacitaciones centralizadas por el equipo implementador de la Oficina General de Estadística e Informática (OGEI) del Ministerio de Salud del Perú en los hospitales II-1 y II-2. Estas capacitaciones estuvieron dirigidas a los médicos y obstetras de los servicios de atención materna. En esta etapa se capacitó a un grupo capacitador local responsable de replicar la implementación en cascada en los establecimientos de salud de la dirección de salud que realizan la atención de partos.

Durante la fase de supervisión se evaluó el cumplimiento de los requerimientos técnicos de cada establecimiento de salud seleccionado en relación a la implementación del Sistema de Información de Nacimientos. El equipo 
local, además de dar el primer nivel de soporte, fue el encargado del monitoreo de las incidencias en el proceso de implementación. Asimismo, la OGEI brindó capacitaciones técnicas, monitorizó y evaluó los procesos implicados dentro del Sistema de Información de Nacimientos en coordinación con el RENIEC.

\section{EVALUACIÓN INICIAL DEL SISTEMA DE INFORMACIÓN DE NACIMIENTOS EN LÍNEA}

En un año de funcionamiento, periodo comprendido entre el 6 de marzo de 2012 al 5 de marzo de 2013, el sistema de Registro del Certificado de Nacido Vivo en Línea ha sido implementado en 114 establecimientos de salud de 21 regiones, donde se atienden partos (tanto públicos como privados); con una mayor concentración (40\%) en Lima y Callao. Durante este periodo, el Registro del Certificado de Nacido Vivo en Línea informó 103917 recién nacidos vivos. De ellos, el $84 \%$ provienen de las regiones de Lima, Callao, Cusco y Piura; el 47,4\%, de registros provinieron de hospitales II-1; el 27,2\%, de centros materno infantiles; el 15,8\%, de hospitales nacionales III-1 y III-2; y el 9,6\%, de registros provinieron de establecimientos de salud privados.

En la Tabla 1 se muestra el número de los establecimientos de salud por regiones, en los cuales se implementó el sistema de Registro del Certificado de Nacido Vivo en Línea durante el periodo marzo de 2012 a marzo de 2013. Del total de estos establecimientos

Tabla 1. Establecimientos implementados por regiones: marzo 2012 - marzo 2013

\begin{tabular}{lr}
\hline Regiones & $\mathbf{N} .^{\circ}(\%)$ \\
\hline Lima & $43(38)$ \\
Ayacucho & $12(10)$ \\
Amazonas & $8(7)$ \\
Cusco & $5(4)$ \\
La Libertad & $5(4)$ \\
Apurímac & $4(3,5)$ \\
Huánuco & $4(3,5)$ \\
Piura & $4(3,5)$ \\
Tumbes & $4(3,5)$ \\
Arequipa & $3(2,6)$ \\
Callao & $3(2,6)$ \\
Ica & $3(2,6)$ \\
Lambayeque & $3(2,6)$ \\
Puno & $3(2,6)$ \\
Madre de Dios & $2(2)$ \\
Moquegua & $2(2)$ \\
Ucayali & $2(2)$ \\
Cajamarca & $1(1)$ \\
Huancavelica & $1(1)$ \\
San Martín & $1(1)$ \\
Tacna & $1(1)$ \\
\hline
\end{tabular}

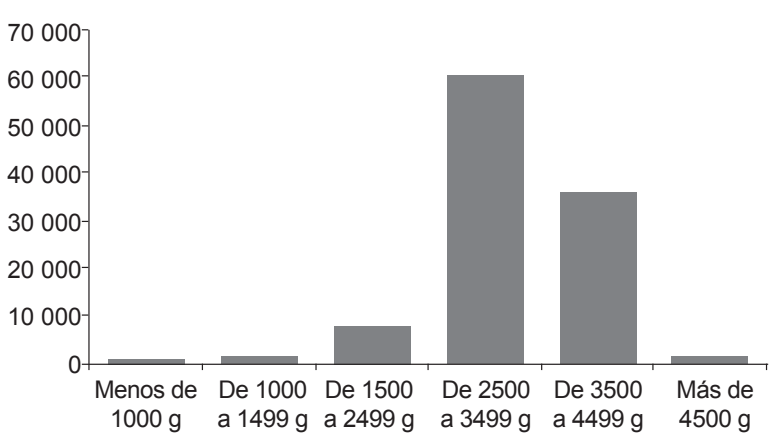

Figura 1. Nacidos vivos según el peso al nacer, 2012-2013

de salud, el 88,6\% (101/114) pertenecen al Ministerio de Salud; el 9,6\% (11/114), al sector privado; y 1,8\% (2/114), a EsSalud y a las Fuerzas Armadas.

Un ejemplo de las bondades de este nuevo sistema para la generación de información de manera rápida, oportuna y eficaz es que, para abril de 2013 se conocía, además del total de recién nacidos registrados en el sistema de Registro del Certificado de Nacido Vivo en Línea, datos como que el 51,5\% (53 560/103 917) fue del sexo masculino; que el 5,4\% (5584) de los nacidos vivos proviene de madres adolescentes (11-17 años); el 6,9\% (7135/103 907) presentó un peso por debajo de 2500 g (Figura 1); el 92,6\% (96 158/ 103 917) de los nacidos vivos fueron nacimientos a término ( $>37$ semanas), y que el $7,4 \%$ (7585/103 917) fueron nacimientos de 36 semanas a menos. Datos con los cuales se pudo realizar un análisis bivariado, el cual mostró que las madres adolescentes tienen un mayor riesgo relativo de presentar embarazos pretérminos (<37 semanas), recién nacidos con bajo puntaje apgar a los 5 minutos (<7) y bajo peso al nacer $(<2500 \mathrm{~g})$, en comparación con las madres adultas (> 18 años); todo ello con un nivel de confianza fijado al $95 \%$ y un valor $p \leq 0,05$ como significancia estadística (Tabla 2).

\section{ALGUNAS REFLEXIONES FINALES}

Un sistema de registro de hechos vitales efectivo es aquel sistema que proporciona datos procesables que pueden utilizarse para mejorar la calidad de los servicios de salud, mejorar el análisis y la presentación de las estadísticas vitales; y, más importante aun, lo suficientemente robusto como para interoperar con otros sistemas como los sistemas de historias clínicas electrónicas, y otros registros relevantes de salud pública ${ }^{(9)}$.

Es importante considerar que el recojo de información de las variables demográficas, del recién nacido y de 
Tabla 2. Factores de riesgo en madres adolescentes, marzo 2012 - febrero 2013

\begin{tabular}{|c|c|c|c|c|c|}
\hline Factores de riesgo & $\begin{array}{c}\begin{array}{c}\text { Adolescentes } \\
\text { N. }\end{array}{ }^{\circ}(\%) \\
\end{array}$ & $\begin{array}{c}\text { Adultas } \\
\mathrm{N} .^{\circ}(\%)\end{array}$ & $\mathbf{R R}$ & IC $95 \%$ & p \\
\hline \multicolumn{6}{|l|}{ Peso recién nacido } \\
\hline$<2500 \mathrm{~g}$ & $472(6,6)$ & $6663(93,4)$ & 1,25 & $1,14-1,37$ & $<0,01$ \\
\hline$\geq 2500 \mathrm{~g}$ & $5112(5,3)$ & $91476(94,7)$ & & & \\
\hline \multicolumn{6}{|c|}{ Puntaje APGAR a los $5 \mathrm{~min}$} \\
\hline$\leq 7$ & $91(7,7)$ & $1089(92,3)$ & 1,49 & $1,22-1,82$ & $<0,01$ \\
\hline 8 a 10 & $3138(5,2)$ & $57396(94,8)$ & & & \\
\hline \multicolumn{6}{|l|}{ Edad gestacional } \\
\hline$<37$ semanas & $484(6,4)$ & $7101(93,6)$ & 1,19 & $1,09-1,31$ & $<0,01$ \\
\hline$\geq 37$ semanas & $5136(5,3)$ & $91022(94,7)$ & & & \\
\hline
\end{tabular}

* Se consideró como madre adolescente a todas aquellas mujeres $\leq 18$ años de edad

la madre son las mismas tanto en el sistema en papel como en el sistema en línea. Sin embargo, el nuevo registro en línea incorpora además el Código Único de Identificación y añade al certificado generado un código de barras. Todo ello permite facilitar y optimizar el correcto registro de hechos vitales en el RENIEC, logrando posteriormente la obtención del DNI del niño, que permite garantizar el derecho a la identidad, uno de los derechos fundamentales de la persona.

A través del análisis de los datos del registro en línea, los subgrupos de población con necesidades específicas de salud o factores de riesgo pueden ser identificados y así, los programas e intervenciones en salud pueden ser adaptados a circunstancias particulares y los sistemas de vigilancia epidemiológica pueden ser activados oportunamente ${ }^{(5)}$. Por ejemplo, el análisis de los registros de nacimientos 2012-2013 muestra que $7 \%$ de los recién nacidos presentaron bajo peso, por tanto, el sistema de vigilancia epidemiológica puede ser fortalecido y activado oportunamente de tal manera que los servicios de salud respondan adecuadamente.

Se debe mencionar, que no somos ni el primero ni el único país que ha implementado este tipo de registros. Por el contrario, muchos países utilizan los registros de hechos vitales como una fuente continua y completa de datos para la planificación de programas de salud, la formulación de políticas de salud, para evaluación de programas, e investigación (10,11). Por ejemplo, en Dinamarca, los datos de los registros de hechos vitales se utilizan para investigación epidemiológica ${ }^{(12)}$ y en Washington, Estados Unidos, los datos de hechos vitales se utilizan para identificar grupos de alto riesgo, para priorizar estrategias de prevención de enfermedades, y planificar programas de promoción a la salud ${ }^{(13)}$.

Existen desafíos que son necesarios considerar para una mejor implementación del sistema de información de nacimientos en línea, como son: una adecuada infraestructura que incluya apropiada conectividad a Internet de parte de los establecimientos de salud y terminales computarizados conectados a una impresora. Además, es necesario garantizar la interoperabilidad para que el sistema de nacimientos en línea se integre con los demás subsistemas y aplicativos del sector salud ${ }^{(14)}$. Por otro lado, la capacitación de los recursos humanos es fundamental, no solo en temas de ofimática sino también en las áreas de sistemas de información en salud, gestión de conocimiento y manejo del cambio ${ }^{(9)}$. Queda por último mencionar que en nuestro país todavía existen muchos partos que ocurren fuera de los establecimientos de salud.

Es necesario, además, mencionar que existen algunas potencialidades de este sistema que se pueden fortalecer. Tal como se ha descrito en países como Estados Unidos ${ }^{(15)}$, este sistema de información de nacimientos se puede complementar con servicios de telemedicina a través de teleconsultas y telediagnósticos, sobre todo para pacientes en alto riesgo.

Para concluir, se debe tener presente que durante el período evaluado ( 6 de marzo de 2012 al 5 de marzo de 2013) el Sistema de Información de Nacimientos en Línea registró aproximadamente a un tercio del total de nacimientos del país, proporcionando información precisa, oportuna y de calidad sobre los nacimientos. Por lo que consideramos que el Sistema de Información de Nacimientos en Línea se constituye en un eje central del sistema de información en salud, el cual permite obtener estadísticas en tiempo real para una adecuada y oportuna toma de decisiones en salud pública.

Contribuciones de autoría: WHC tuvo la idea y concepción del estudio, WHC, y KP recolectaron los datos, WHC, KP, y $\mathrm{ML}$ analizaron e interpretaron los datos, WHC redactó el manuscrito, WHC, KP y ML hicieron la revisión crítica, todos los autores aprobaron la versión final del artículo.

Fuentes de financiamiento: autofinanciado.

Conflictos de interés: WHC es el director general de la Oficina General de Estadística e Informática (OGEI) del Ministerio de Salud (MINSA). KP es la directora ejecutiva de la Oficina de Estadística de la OGEI del MINSA y ML es jefe de equipo del Área de Producción Estadística de la Oficina de Estadística de la OGEI del MINSA. 


\section{REFERENCIAS BIBLIOGRÁFICAS}

1. Murray C J. Towards good practice for health statistics: lessons from the Millennium Development Goal health indicators. Lancet. 2007;369(9564):862-73.

2. Setel PW, Macfarlane SB, Szreter S, Mikkelsen L, Jha P, Stout S, AbouZahr C. A scandal of invisibility: making everyone count by counting everyone. Lancet. 2007;370(9598):1569-77.

3. United Nations Children's Fund (UNICEF). The 'rights' start to life. A statistical analysis of birth registration. New York: United Nations Children's Fund; 2005.

4. Hinman AR, Atkinson D, Diehn TN, Eichwald J, Heberer J, Hoyle T, et al. Principles and core functions of integrated child health information systems. J Public Health Manag Pract. 2004;Suppl:S52-6.

5. WalkerN,BryceJ,BlackRE. Interpreting health statistics for policymaking: the story behind the headlines. Lancet. 2007;369(9565):956-63.

6. Evans T, Stansfield S. Health information in the new millennium: a gathering storm? Bull World Health Organ. 2003;81(12):856.

7. Stansfield SS, Walsh J, Prata N, Evans T. Information to improve decision making for health. In: Jamison DT,
Breman JG, Measham AR, et al. Disease control priorities in developing countries, 2nd ed. Washington DC: World Bank; 2006. p. 1017-30.

8. Perú, Ministerio de Salud. Resolución Ministerial 148-2012/MINSA que aprueba la Directiva Administrativa $\mathrm{N}^{\circ}$ 190-MINSA/OGEI-V01 que establece el Procedimiento para el Registro del Certificado de Nacido Vivo. Disponible en: ftp://ftp2.minsa.gob. pe/normaslegales/2012_/RM1482012-MINSA-OLD.PDF

9. Curioso WH. Salud electrónica en el Perú: un tema emergente. En: Fernández A, Oviedo E (eds). Salud electrónica en América Latina y el Caribe: Avances y desafíos. Santiago de Chile: Naciones Unidas; 2010. p.113-21.

10. Rothwell CJ. Reengineering Vital Registration and Statistics Systems for the United States. Prev Chronic Dis. 2004;1(4):A03.

11. Devlin HM, Desai J, Walaszek A. Reviewing performance of birth certificate and hospital discharge data to identify births complicated by maternal diabetes. Matern Child Health J. 2009;13(5):660-6.

12. Pedersen CB. The Danish Civil Registration System. Scand J Public
Health. 2011;39(7 Suppl):22-5.

13. Washington State, Department of Health. Vital Statistics Data [Internet]. 13. Washington, DC: Department of Health Internet; c2013 [citado el 8 de marzo del 2013]. Disponible en: http:// www.doh.wa.gov/DataandStatisticalReports/VitalStatisticsData.aspx

14. Chapman DA, Ford N, Tlusty S, BodurthaJN. Evolution of an integrated public health surveillance system. J Registry Manag. 2011;38(1):15-23.

15. Gray JE, Safran C, Davis RB, PompilioWeitzner G, Stewart JE, Zaccagnini L, et al. Baby CareLink: using the internet and telemedicine to improve care for high-risk infants. Pediatrics. 2000;106(6):1318-24.

Correspondencia: Walter H. Curioso Dirección: Ministerio de Salud del Perú, Oficina General de Estadistica e Informática, Av. Salaverry 801 Jesús María. Lima, Perú Teléfono: (511) 3156600

Correoelectrónico:wcurioso@minsa.gob.pe 